\title{
Engineering of oleaginous organisms for lipid
}

\section{production}

Jennifer Levering, Jared Broddrick, Karsten Zengler

Department of Bioengineering, University of California San Diego, La Jolla, CA 92093, USA

Corresponding author: Karsten Zengler (kzengler@ucsd.edu)

Author email addresses:

Jennifer Levering: jlevering@ucsd.edu

Jared Broddrick: jtb@ucsd.edu 


\section{Abstract}

Phototrophs are attractive candidates for commercial lipid production. Lipid biosynthetic pathways in these organisms have been largely characterized but the mechanisms partitioning resources towards storage lipids are poorly understood. One promising strategy to study and enhance biomass lipid bioproduction in oleaginous microorganisms is to combine genome-scale metabolic modeling and genetic and metabolic engineering. Here we describe recent advances in in vitro, in vivo, and in silico manipulations of phototrophic metabolism that increase total lipid content or redirect lipid production towards more favorable products such as polyunsaturated fatty acids used as nutritional supplements or in biofuel production.

\section{Introduction}

Excessive demands by modern society have been depleting nature's resources over the past centuries. Exploring and developing new sustainable resources to counter increasing consumption has therefore been the focus of current research efforts in the academic and private sector. The emphasis has partly been on investigating the use of phototrophic organisms that are able to fix carbon dioxide by utilizing light energy and produce energy-dense products. Lipids derived from photosynthetic organisms have therefore been explored to augment existing sources for energy and nutrition $[1,2]$. Efforts for generating lipid-derived products at the industrial scale have been predominantly centered around microalgae and peripherally around oleaginous yeast. 
While the biosynthetic pathways for fatty acid and lipid metabolism in these organisms are fairly well understood, how organisms prioritize carbon and energy towards those pathways is still being explored. As a result, there has been a surge in the attempts to understand and subsequently manipulate the cellular processes responsible for allocating resources for the production of lipid biomass. Carbon partitioning is the natural method by which an organism directs resources into various metabolic processes. In phototrophs, this process is often at odds with the accumulation of engineered targets, i. e. desired lipids, with a bias towards non-desirable carbohydrate biosynthesis [3]. Strategies for maximizing lipid biomass include engineering the cellular metabolism towards a desired biomass composition and taking advantage of natural processes of enhancing carbon towards lipid biomass such as unfavorable growth conditions. These conditions lead to environmental and nutrient stress induced growth arrests in photosynthetic microorganisms which usually result in an accumulation of storage metabolites, including lipids [4]. However, the organism's inherent partitioning bias between lipids and carbohydrates remains intact. Thus, large scale screens to identify organisms with inherently favorable partitioning has been a common method of choice $[5,6]$. Increasing the total resource accumulation is another strategy that has been explored since excess nutrient supply is generally diverted to storage molecules. Mixotrophic cultures, where a $\mathrm{CO}_{2}$-fixing phototroph is provided additional resources in the form of an organic carbon source, often results in higher biomass yields [7]. However, the benefit in high yield has to outweigh the cost of providing additional carbon. To circumvent this challenge, designing symbiotic co-cultures with organic carbon-producing phototrophs and organic carbon-utilizing oleaginous heterotrophs is a 
promising strategy for advanced lipid biomass production [8]. Finally, genome-scale modeling of cellular metabolism can be used as a framework for understanding intracellular carbon partitioning and to create optimized, at times non-intuitive, strategies for maximizing target compounds.

\section{Challenges in lipid-producing microorganisms}

Organisms currently being investigated for the production of biofuels and lipids include plants, unicellular phototrophs, and oleaginous heterotrophs. The use of land plants for the production of bioethanol either directly from the plant storage sugars or via processing agricultural waste are options that have been aggressively investigated [9]. For lipid-derived sources, edible and non-edible oilseed crops are being explored [10]. The challenges associated with these sources are centered on the food vs. fuel conundrum [11] and the environmental impact [12]. These challenges are constantly cited as the driver for choosing a microbial-based platform for lipid products. Unicellular phototrophs, such as bacteria and microalgae, exhibit high photosynthetic efficiency, can be grown on non-arable land with minimal inputs $\left(\mathrm{CO}_{2}\right.$, light, and vitamins), and naturally produce desired products, such as nutritional omega-3 fatty acids. However, most industrial-scale applications for biofuels are currently limited by the challenge of generating biomass along with the economics of scale: bioreactors are inefficient and open (outdoor) cultures are complex [13]. The economics become more feasible with the concurrent generation of high-value products such as omega-3 nutritional supplements, carotenoid-based antioxidants, as well as pigments [14-16]. The success 
of microbial-based lipid biomass platforms, however, is highly dependent on our understanding of metabolic and regulatory bottlenecks.

\section{Lipid biosynthetic pathways}

Fatty acid and lipid biosynthetic pathways in plants, unicellular phototrophs, and oleaginous yeast have been well studied (for recent reviews see for example [17-20]). A visual overview of these pathways can be found in Figure 1. Acetyl-CoA carboxylase (ACCase) catalyzed generation of malonyl-CoA is the first committed step of fatty acid biosynthesis; Huerliman and Heimann have published a comprehensive review of algal ACCases [21]. Fatty acid de novo biosynthesis occurs in the chloroplast of phototrophs or in the cytosol of yeast with the acyl-activating moiety differing between the compartments (acyl carrier protein vs. CoA respectively). The fatty chain length is extended two carbons at a time involving four enzymes that make up the fatty acid synthase complex. In phototrophs, a cytosolic set of fatty acid synthase enzymes can extend the chain length to form very long $(\mathrm{C} 20-\mathrm{C} 24)$ saturated or monounsaturated fatty acids. While polyunsaturated fatty acids (PUFAs) of the $\mathrm{C} 16$ and $\mathrm{C} 18$ variety are synthesized in the chloroplast, very long chain omega-3 PUFA biosynthesis occurs between the endoplasmic reticulum and the cytosol. Acyl-editing, a well orchestrated cycle of fatty acid elongation and desaturation, synthesizes high-value omega-3 fatty acids and is largely responsible for their incorporation into triacylglycerols (TAGs) [22]. Lipid biogenesis begins by siphoning triose phosphate away from central carbon metabolism, reducing the oxo functional group, and acylation with activated fatty acids to form phosphatidic acid. Phosphatidic acid is the starting point for the biogenesis of 
the vast array of membrane lipids (phosphatidylcholine, diacylglycerol, monogalatosyldiacylglycerol, etc) as well as TAGs. Photosynthetic membrane biogenesis is a chloroplastic process with specific fatty acid requirements. Nonphotosynthetic membrane synthesis occurs in the cytosol and endoplasmic reticulum, requiring chloroplast generated fatty acids in phototrophs or cytosol generated fatty acids in heterotrophs. The metabolic input required for TAG biosynthesis requires either an excess of carbon and energy or the conversion of existing resources. For the latter, remodeling of existing membrane lipids into TAGs accompanies various types of physiological changes or stress including changes in light, $\mathrm{pH}$, temperature, and nutrient [23-28]. While the biosynthetic pathways have been largely characterized, the regulatory mechanisms that partition resources towards storage lipids are still poorly understood, thus presenting an unexplored resource for design strategies.

\section{Strategies for lipid production}

Taking advantage of our current knowledge about lipid biosynthetic pathways, several strategies have been exploited to optimize lipid production in photosynthetic organisms ranging from exposure to environmental and nutrient stress to metabolic engineering using transgene expression as well as co-culturing and in silico investigations of flux distributions. Figure 2 gives a schematic overview of the discussed strategies for enhanced lipid production in phototrophs.

\section{Inherent carbon partitioning}


Oleaginous microorganisms naturally exhibit differential carbon partitioning between carbohydrates and lipids. Liang and Jiang reviewed the potential of oleaginous microorganisms for biodiesel production including bacteria, fungi, microalgae, and yeast and collected the total biomass and biomass lipid content for selected microalgae [29]. Based on this data, Chaetoceros gracilis produces the highest lipids per biomass followed by Tetraselmis tetrathele, Nannochloropsis oculata, Chaetoceros muelleri, and Chlorella protothecoides as shown in Figure 3. Their lipid content per dry weight ranges from $23 \%$ for Nannochloropsis to $60 \%$ for Chaetoceros. Among the other investigated organisms are microalgae with higher lipid content but lower total biomass resulting in lower lipid productivity such as Chlorella $s p$. with a lipid content of $52 \%$ and a biomass yield 15 times lower compared to Chaetoceros. Thalassiosira weissflogii and Cylotella cryptica showed favorable lipid accumulation in a comprehensive screen of marine diatoms [6]. A screen of 69 strains of oleaginous yeast grown under various culture conditions identified a variety of candidates with lipid content comparable to that of photosynthetic microorganisms [30]. The most productive oleaginous yeast genus (Rhodosporidium) had a biomass yield 1-2 orders of magnitude higher than the most attractive diatom candidates with similar total lipid composition [30]. The dependence of growth rate and carbon fixation on light in phototrophs is likely the source of this disparity. Yet, supplementing phototrophs with organic carbon increased the biomass yield by approximately 3-fold in different Chlorella strains and Nannochloropsis [7]. In a separate study, Chlorella sorokiniana cultured under mixotrophic and pure heterotrophic conditions were able to achieve biomass yields comparable to yeast [31]. To offset the additional cost of organic carbon supplementation, wastewater and biodiesel waste (i.e. 
glycerol) have been successfully used in microalgae [32,33] and oleaginous yeast cultivation [34-36].

Environmental or nutrient stress in microalgae induces a shift towards nutrient storage [4]. Lipid accumulation and fatty acid redistribution during nutrient deprivation has been extensively studied in diatoms [37-42], microalgae [43-46] and oleaginous yeast [35]. Studies in Chlamydomonas reinhardtii [44,46] and Phaeodactylum tricornutum [42] showed degradation of the photosynthetic apparatus accompanies stress-induced lipid accumulation in phototrophs. This effect can also be artificially initiated at faster time scales using small molecules as shown in Chlamydomonas reinhardtii [47]. Thus, the accumulation of TAGs during stress is a reallocation of existing protein and lipid biomass allocated to the photosynthetic apparatus; biomass that is also partitioned into carbohydrates based on the organism's carbon allocation biases. Metabolic flux analysis of Chlorella protothecoides [48] and Trichosporon cutaneum [49] experimentally determined the carbon partitioning of a microalgae and oleaginous yeast respectively.

\section{Manipulation of lipid metabolism using metabolic engineering}

Recent advances in metabolic engineering tools for photosynthetic organisms demonstrated that they are attractive candidates for industrially-relevant lipid production. Transgenic expression of high-efficiency desaturases and elongases was, for example, applied to enhance the production of PUFAs. Hamilton et al. optimized the omega-3 content in $P$. tricornutum by expressing a $\Delta 5$-elongase gene from the green alga Ostreococcus tauri [50]. Co-expression of microalgal $\Delta 5$-elongase and $\Delta 4$ - 
desaturase into the oleaginous yeast Pichia pastoris led to docosahexaenoic acid production in the transgenic yeast which naturally cannot produce PUFAs longer than C18 linoleic and $\alpha$-linolenic acid [51].

Due to the fact that fatty acid synthesis is tightly regulated in most organisms, overexpression of genes involved in fatty acid production can lead to reduced flux towards TAG production. To minimize regulatory effects such as feedback inhibition, Tai and Stephanopoulus co-overexpressed the genes corresponding to the first and last step in the lipid synthesis pathway in the oleaginous yeast Yarrowia lipolytica [52]. The biomass lipid content was increased more than 4 -fold to about $41 \%$ with a slightly changed fatty acid profile. Overexpression can also be used to change the fatty acid profile as shown in $P$. tricornutum [53]. The overexpression of glycerol-3-phosphate dehydrogenase resulted in a shift towards neutral fatty acid production. Using antisense and interfering RNA, Trentacoste and coworkers knocked-down genes involved in lipid catabolism in the diatom Thalassiosira pseudonana to increase lipid accumulation without compromising growth [54]. Another important step towards the exploitation of diatoms as biofuel producers is a targeted genome modification using, for example, meganucleases and transcription activator-like effector nucleases (TALENs) [55]. The authors were able to engineer seven genes in the lipid metabolism of $P$. tricornutum resulting in a $45 \%$ increase in TAG production. An alternative, RNA-dependent targeted genome engineering technology is the CRISPR/Cas9 system that has been shown to be efficient in a wide range of organisms [56], including industrial relevant Saccharomyces cerevisiae strains [57] as well as Escherichia coli [58]. Compared to TALENs, the CRISPR/Cas system promises to be easier to design and use but has not 
been implemented in microalgae yet. However, we anticipate this technology to be applied for lipid metabolism engineering due to its promising features.

Recently, Karas and coworkers demonstrated the transfer of genetic material from $E$. coli to the diatoms $P$. tricornutum and T. pseudonana using conjugation [59]. This method can provide for the efficient transfer of large amounts of genetic material such as entire pathways and will facilitate and accelerate genome manipulations in these organisms.

Often, the aforementioned metabolic engineering approaches result in strains unable to dynamically regulate gene expression [60]. Xu and coworkers significantly improved fatty acids production by rewiring an existing transcriptional regulator to dynamically control expressions of genes involved in synthesis and degradation of malonyl-CoA in Escherichia coli. Due to lacking knowledge about the regulatory networks of oleaginous microorganisms this engineering approach was not much exploited yet.

\section{Enhanced lipid production through co-culturing}

Another strategy to improve lipid production involves the exploitation of symbiotic relationships between microorganisms with at least one photosynthetic species. So far, organisms exploited for these applications include fungi, microalgae, and phototrophic and heterotrophic bacteria [8]. Compared to monocultures, co-cultures can demonstrate enhanced growth rates as well as higher total biomass and lipid content since both partners contribute to biomass production and composition. Thus, the choice of the partners influences the overall fatty acid composition. Lipids obtained from a co-culture of Trichosporonoides spathulata and Chlorella vulgaris, for example, were mainly 
composed of palmitic, oleic, and stearic acid and showed a significantly higher content of saturated fatty acids than those of the monocultures [61].

When partnering up yeast and microalgae, yeast provides additional $\mathrm{CO}_{2}$ to the algae which in return generates $\mathrm{O}_{2}$ for the yeast [61]. Partnering microalgae and fungi can also enhance the harvesting process which requires a high amount of energy and can be very costly [62]. Wrede and co-authors studied fungal-assisted flocculation for different algae, including marine microalgae, for this purpose. Another possibility for facilitating the harvesting process is the encapsulation of the co-cultured organisms in alginate gel beads as demonstrated for Trichosporonoides spathulata and Chlorella vulgaris [61]. Although biomass and lipid production slightly decreased compared to free cell cultivation, harvesting was facilitated and less costly. Most studies focus on artificial co-cultures although native communities have been shown to be viable feedstocks for biofuels and bioproducts, too $[63,64]$.

\section{Modeling}

Genome-scale metabolic models have proven to be powerful tools to understand and predict genotype-phenotype relationships. Flux balance analysis [65] can be used to predict intracellular flux distributions with good accuracy [66] allowing for in silico predictions of carbon partitioning. Elucidation of intracellular flux in silico gives insight into carbon partitioning and can be used to generate targets for metabolic engineering. This has successfully been deployed by using a genome-scale reconstruction of the oleaginous yeast Mortierella alpina to optimize the industrial production process of PUFAs [67]. 
Several mathematical optimization strategies are available that facilitate the identification of targeted genetic manipulation strategies in silico. These tools allow for the redesign of metabolism while simulating the impact on a specified objective. Additionally, computational approaches reveal non-obvious redesign strategies by suggesting manipulations in pathways distant from the studied target [68]. Different genetic manipulations can be identified using the available computational approaches. Some of these procedures, including OptKnock, can be used to identify gene deletions resulting in the overproduction of a desired metabolite while ensuring biomass production [69]. Another class of computational approaches for metabolic engineering can be used to identify a combination of gene deletions, down-regulations and overexpression, for example OptForce [70]. This method was successfully used to optimize the production of fatty acids with a chain length of six to 16 carbons in $E$. coli [68]. Other available approaches for metabolic engineering can be used for strain design and identify non-native pathway additions. For example, Ip and coworkers applied in silico metabolic engineering to predict the effects of gene overexpression and insertion of heterologous pathways on free fatty acid production and experimentally validated their findings [71]. Currently available procedures were recently reviewed in $[72,73]$.

Genome-scale metabolic modeling can also be exploited to understand the complex interactions between microorganisms in natural and artificial communities. So far, community modeling has not been utilized to optimize the lipid content and composition in co-cultures but was successfully applied to simulate other aspects of microbial communities, such as the interactions between the partners [74]. 


\section{Conclusion}

Microalgae and oleaginous yeast accumulate a large part of their dry weight in lipids. However, to use these organisms for industrial applications, lipid biomass yield and productivity need to be further optimized. In this review, we provided an overview on promising strategies to enhance lipid production. Unfavorable growth conditions such as environmental and nutrient stress enhance lipid production while slowing down the growth rate. Recent advances in genetic engineering and manipulation of photosynthetic organisms promise to aid the construction of biofuel production strains without compromising growth. Genome-scale modeling promises to aid the identification of potential phototroph lipid producers but also to facilitate and speed up the metabolic engineering of these organisms. Additionally, genome-scale modeling facilitates gaining a more detailed understanding of the phototrophs metabolic capabilities and regulation which is essential for engineering these organisms. 


\section{Figures}

Figure 1: Lipid biosynthesis pathway in phototrophs and heterotrophs. Generic representation of fatty acid and lipid biosynthetic pathways in phototrophic and heterotrophic organisms. The large colored arrows indicate branch points for carbon partitioning between energy generation and storage in the form of carbohydrates or lipids. Abbreviations: Ac-CoA, acetyl-coenzyme A; ACCase, acetyl-CoA carboxylase; DAG, diacylglycerol; DHAP, dihydroxyacetone phosphate; FA, fatty acid (C-number is the carbon chain length); G3P, glyceraldehyde-3-phosphate; Gly-3-P, glycerol-3phosphate; Mal-CoA, malonyl-coenzyme A; PA, phosphatidic acid; TAG, triacylglycerol.

Figure 2: Strategies employed to enhance lipid production in photosynthetic organisms. Lipid biomass production can be enhanced using different strategies such as redirecting carbon toward lipid production using nutrient or environmental stress. Genome manipulations promise a favorable lipid biomass production while not slowing down growth rate. Due to the fact that both partners contribute to the biomass content, co-culturing can be used to enhance lipid productivity. Genome-scale models can facilitate and guide efforts in all shown strategies.

Figure 3: Lipid biosynthesis in phototrophs. Lipid productivity (mg/L/day) against biomass productivity ( $\mathrm{g} / \mathrm{L} /$ day) of selected microalgae based on data from [29]. Circle sizes are scaled by lipid content. Lipid content and biomass productivity corresponding to the higher lipid productivity were selected in case a range was given. 


\section{Acknowledgements}

This material is based upon work supported by the U.S. Department of Energy, Office of Science, Office of Biological and Environmental Research under Award Number DE-

SC0008701 and the Scientific Biological Systems Science Division under Award Numbers DE-SC0012586, DE-SC0012658. 


\section{References and recommended reading}

Papers of particular interest, published within the period of review, have been highlighted as:

-of special interest

• of outstanding interest

•[30] Sitepu (2013)

Large-scale screen of inherent carbon partitioning in oleaginous yeast. Identified species with high biomass yield and a large lipid fraction. Valuable insight into the biodiversity of carbon partitioning in yeast.

•[41] Abida et al (2014)

In depth lipid analysis of nitrogen and phosphorous starvation. Elucidated a two step transition in phosphorus limitation as well as characterizing the fatty acid and major lipid class distributions.

••[42] Levitan et al (2015)

Comprehensive characterization of proteome and lipid remodeling following nitrogen starvation. Revealed almost half the proteome is degraded and re-partitioned to the nitrogen assimilation mechanism and storage compounds.

••[44] Schmollinger (2014)

Systems-level analysis of the transition to nitrogen starvation at the transcript and proteome level. A nitrogen conservation mechanism is initiated that reduces expression of nitrogen rich proteins; mainly elements of photosynthesis.

•[54] Trentacoste et al (2013) 
Targeted knockdown of genes involved in lipid catabolism using RNA interference can enhance lipid accumulation without compromising growth in the diatom Thalassiosira pseudonana.

••[55] Daboussi et al, 2014

A highly efficient method for targeted genome editing in diatoms based on meganucleases and TALE nucleases was developed. The method was used to construct modified Phaeodactylum tricornutum strains with 45-fold enhanced TAG production.

••[59] Karas et al (2015)

Highly efficient conjugation-based method for genetic information transfer from Escherichia coli to diatoms. This system facilitates research in diatoms not only by enabling the characterization of genes but also by allowing introduction of entire pathways into the organism.

•[71] Ip et al., 2014

A constraint-based modeling approach for simulating the effect of a heterologous gene insertion is presented and, together with FBA-based approaches for strain optimization, applied to predict engineering strategies for overproduction of free fatty acids in Escherichia coli. 
1. Liu B, Benning C: Lipid metabolism in microalgae distinguishes itself. Curr. Opin. Biotechnol. 2013, 24:300-309.

2. Lenihan-Geels G, Bishop KS, Ferguson LR: Alternative sources of omega-3 fats: Can we find a sustainable substitute for fish? Nutrients 2013, 5:1301-1315.

3. Melis A: Carbon partitioning in photosynthesis. Curr. Opin. Chem. Biol. 2013, $17: 453-456$.

4. Cheng D, He Q: Assessment of environmental stresses for enhanced microalgal biofuel production - An overview. Front. Energy Res. 2014, 2:26.

5. Breuer G, Lamers PP, Martens DE, Draaisma RB, Wijffels RH: The impact of nitrogen starvation on the dynamics of triacylglycerol accumulation in nine microalgae strains. Bioresour. Technol. 2012, 124:217-226.

6. d'Ippolito G, Sardo A, Paris D, Vella FM, Adelfi MG, Botte P, Gallo C, Fontana A: Potential of lipid metabolism in marine diatoms for biofuel production. Biotechnol. Biofuels 2015, 8:28.

7. Cheirsilp B, Torpee S: Enhanced growth and lipid production of microalgae under mixotrophic culture condition: Effect of light intensity, glucose concentration and fed-batch cultivation. Bioresour. Technol. 2012, 110:510516.

8. Santos CA, Reis A: Microalgal symbiosis in biotechnology. Appl. Microbiol. Biotechnol. 2014, 98:5839-5846.

9. Sarkar N, Ghosh SK, Bannerjee S, Aikat K: Bioethanol production from agricultural wastes: An overview. Renew. Energy 2012, 37:19-27.

10. Atabani AE, Silitonga AS, Ong HC, Mahlia TMI, Masjuki HH, Badruddin IA, Fayaz H: Non-edible vegetable oils: A critical evaluation of oil extraction, fatty acid compositions, biodiesel production, characteristics, engine performance and emissions production. Renew. Sustain. Energy Rev. 2013, 18:211-245.

11. Condon N, Klemick H, Wolverton A: Impacts of ethanol policy on corn prices: A review and meta-analysis of recent evidence. Food Policy 2015, 51:63-73.

12. Wright CK, Wimberly MC: Recent land use change in the Western Corn Belt threatens grasslands and wetlands. Proc. Natl. Acad. Sci. U. S. A. 2013, 110:4134-4139.

13. Apel AC, Weuster-Botz D: Engineering solutions for open microalgae mass cultivation and realistic indoor simulation of outdoor environments. Bioprocess Biosyst. Eng. 2015, 38:995-1008. 
14. Wang H-MD, Chen C-C, Huynh P, Chang J-S: Exploring the potential of using algae in cosmetics. Bioresour. Technol. 2015, 184:355-362.

15. Heydarizadeh P, Poirier I, Loizeau D, Ulmann L, Mimouni V, Schoefs B, Bertrand M: Plastids of marine phytoplankton produce bioactive pigments and lipids. Mar. Drugs 2013, 11:3425-3471.

16. Vinayak V, Manoylov K, Gateau H, Blanckaert V, Hérault J, Pencréac'h G, Marchand J, Gordon R, Schoefs B: Diatom milking: A review and new approaches. Mar. Drugs 2015, 13:2629-2665.

17. Li-Beisson Y, Shorrosh B, Beisson F, Andersson MX, Arondel V, Bates PD, Baud S, Bird D, DeBono A, Durrett TP, et al.: Acyl-lipid metabolism. Arab. B. 2013, 11:e0161.

18. Mühlroth A, Li K, Røkke G, Winge $P$, Olsen $Y$, Hohmann-Marriott MF, Vadstein $O$, Bones AM: Pathways of lipid metabolism in marine algae, co-expression network, bottlenecks and candidate genes for enhanced production of EPA and DHA in species of chromista. Mar. Drugs 2013, 11:4662-4697.

19. Johnson X, Alric J: Central carbon metabolism and electron transport in Chlamydomonas reinhardtii: Metabolic constraints for carbon partitioning between oil and starch. Eukaryot. Cell 2013, 12:776-793.

20. Dulermo T, Nicaud JM: Involvement of the G3P shuttle and beta-oxidation pathway in the control of TAG synthesis and lipid accumulation in Yarrowia lipolytica. Metab. Eng. 2011, 13:482-491.

21. Huerlimann $\mathrm{R}$, Heimann $\mathrm{K}$ : Comprehensive guide to acetyl-carboxylases in algae. Crit. Rev. Biotechnol. 2013, 33:49-65.

22. Bates PD, Fatihi A, Snapp AR, Carlsson AS, Browse J, Lu C: Acyl editing and headgroup exchange are the major mechanisms that direct polyunsaturated fatty acid flux into triacylglycerols. Plant Physiol. 2012, 160:1530-1539.

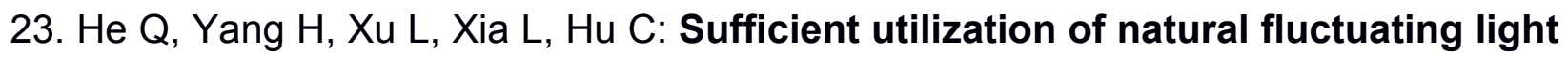
intensity is an effective approach of promoting lipid productivity in oleaginous microalgal cultivation outdoors. Bioresour. Technol. 2015, 180:79-87.

24. Breuer G, Lamers PP, Martens DE, Draaisma RB, Wijffels RH: Effect of light intensity, $\mathrm{pH}$, and temperature on triacylglycerol (TAG) accumulation induced by nitrogen starvation in Scenedesmus obliquus. Bioresour. Technol. 2013, 143:1-9. 
25. Ho SH, Chen $\mathrm{CY}$, Chang JS: Effect of light intensity and nitrogen starvation on $\mathrm{CO}_{2}$ fixation and lipid/carbohydrate production of an indigenous microalga Scenedesmus obliquus CNW-N. Bioresour. Technol. 2012, 113:244-252.

26. Roleda MY, Slocombe SP, Leakey RJG, Day JG, Bell EM, Stanley MS: Effects of temperature and nutrient regimes on biomass and lipid production by six oleaginous microalgae in batch culture employing a two-phase cultivation strategy. Bioresour. Technol. 2013, 129:439-449.

27. Dodson VJ, Mouget JL, Dahmen JL, Leblond JD: The long and short of it: Temperature-dependent modifications of fatty acid chain length and unsaturation in the galactolipid profiles of the diatoms Haslea ostrearia and Phaeodactylum tricornutum. Hydrobiologia 2014, 727:95-107.

28. Montero O, Sánchez-Guijo A, Lubián LM, Martínez-Rodríguez G: Changes in membrane lipids and carotenoids during light acclimation in a marine cyanobacterium Synechococcus sp. J. Biosci. 2012, 37:635-645.

29. Liang $\mathrm{MH}$, Jiang JG: Advancing oleaginous microorganisms to produce lipid via metabolic engineering technology. Prog. Lipid Res. 2013, 52:395-408.

30. Sitepu IR, Sestric R, Ignatia L, Levin D, German JB, Gillies LA, Almada LAG, Boundy-Mills KL: Manipulation of culture conditions alters lipid content and fatty acid profiles of a wide variety of known and new oleaginous yeast species. Bioresour. Technol. 2013, 144:360-369.

31. Li T, Zheng Y, Yu L, Chen S: Mixotrophic cultivation of a Chlorella sorokiniana strain for enhanced biomass and lipid production. Biomass and Bioenergy 2014, 66:204-213.

32. Lizzul AM, Hellier P, Purton S, Baganz F, Ladommatos N, Campos L: Combined remediation and lipid production using Chlorella sorokiniana grown on wastewater and exhaust gases. Bioresour. Technol. 2014, 151:12-18.

33. Cabanelas ITD, Arbib Z, Chinalia F a., Souza CO, Perales J a., Almeida PF, Druzian JI, Nascimento IA: From waste to energy: Microalgae production in wastewater and glycerol. Appl. Energy 2013, 109:283-290.

34. Santamauro F, Whiffin FM, Scott RJ, Chuck CJ: Low-cost lipid production by an oleaginous yeast cultured in non-sterile conditions using model waste resources. Biotechnol. Biofuels 2014, 7:34.

35. Sestric R, Munch G, Cicek N, Sparling R, Levin DB: Growth and neutral lipid synthesis by Yarrowia lipolytica on various carbon substrates under 
nutrient-sufficient and nutrient-limited conditions. Bioresour. Technol. 2014, 164:41-46.

36. Tchakouteu SS, Kalantzi O, Gardeli C, Koutinas AA, Aggelis G, Papanikolaou S: Lipid production by yeasts growing on biodiesel-derived crude glycerol: Strain selection and impact of substrate concentration on the fermentation efficiency. J. Appl. Microbiol. 2015, 118:911-927.

37. Yang Z-K, Niu Y-F, Ma Y-H, Xue J, Zhang M-H, Yang W-D, Liu J-S, Lu S-H, Guan $\mathrm{Y}, \mathrm{Li} \mathrm{H}-\mathrm{Y}$ : Molecular and cellular mechanisms of neutral lipid accumulation in diatom following nitrogen deprivation. Biotechnol. Biofuels 2013, 6:67.

38. Ge F, Huang W, Chen Z, Zhang C, Xiong Q, Bowler C, Yang J, Xu J, Hu H: Methylcrotonyl-CoA carboxylase regulates triacylglycerol accumulation in the model diatom Phaeodactylum tricornutum. Plant Cell 2014, 26:16811697.

39. Mus F, Toussaint J-P, Cooksey KE, Fields MW, Gerlach R, Peyton BM, Carlson RP: Physiological and molecular analysis of carbon source supplementation and $\mathrm{pH}$ stress-induced lipid accumulation in the marine diatom Phaeodactylum tricornutum. Appl. Microbiol. Biotechnol. 2013, 97:3625-3642.

40. Valenzuela J, Carlson RP, Gerlach R, Cooksey K, Peyton BM, Bothner B, Fields MW: Nutrient resupplementation arrests bio-oil accumulation in Phaeodactylum tricornutum. Appl. Microbiol. Biotechnol. 2013, 97:7049-7059.

41. Abida H, Dolch L-J, Meï C, Villanova V, Conte M, Block M a., Finazzi G, Bastien O, Tirichine $\mathrm{L}$, Bowler $\mathrm{C}$, et al.: Membrane glycerolipid remodeling triggered by nitrogen and phosphorus starvation in Phaeodactylum tricornutum. Plant Physiol. 2015, 167:118-136.

42. Levitan O, Dinamarca J, Zelzion E, Lun DS, Guerra LT, Kim MK, Kim J, Van Mooy B a. S, Bhattacharya D, Falkowski PG: Remodeling of intermediate metabolism in the diatom Phaeodactylum tricornutum under nitrogen stress. Proc. Natl. Acad. Sci. U. S. A. 2015, 112:412-417.

43. Urzica El, Vieler A, Hong-Hermesdorf A, Page MD, Casero D, Gallaher SD, Kropat $\mathrm{J}$, Pellegrini M, Benning C, Merchant SS: Remodeling of membrane lipids in iron-starved Chlamydomonas. J. Biol. Chem. 2013, 288:30246-30258.

44. Schmollinger S, Mühlhaus T, Boyle NR, Blaby IK, Casero D, Mettler T, Moseley JL, Kropat J, Sommer F, Strenkert D, et al.: Nitrogen-sparing mechanisms in Chlamydomonas affect the transcriptome, the proteome, and photosynthetic metabolism. Plant Cell 2014, 26:1410-1435. 
45. Martin GJO, Hill DRA, Olmstead ILD, Bergamin A, Shears MJ, Dias DA, Kentish SE, Scales $\mathrm{PJ}$, Botté $\mathrm{CY}$, Callahan DL: Lipid profile remodeling in response to nitrogen deprivation in the microalgae Chlorella sp. (Trebouxiophyceae) and Nannochloropsis sp. (Eustigmatophyceae). PLoS One 2014, 9:e103389.

46. Wei L, Derrien B, Gautier A, Houille-Vernes L, Boulouis A, Saint-Marcoux D, Malnoë A, Rappaport $F$, de Vitry $C$, Vallon $O$, et al.: Nitric oxide-triggered remodeling of chloroplast bioenergetics and thylakoid proteins upon nitrogen starvation in Chlamydomonas reinhardtii. Plant Cell 2014, 26:353-72.

47. Kim H, Jang S, Kim S, Yamaoka Y, Hong D, Song W-Y, Nishida I, Li-Beisson Y, Lee $Y$ : The small molecule fenpropimorph rapidly converts chloroplast membrane lipids to triacylglycerols in Chlamydomonas reinhardtii. Front. Microbiol. 2015, 6:54.

48. Wu C, Xiong W, Dai J, Wu Q: Genome-based metabolic mapping and $13 \mathrm{C}$ flux analysis reveal systematic properties of an oleaginous microalga Chlorella protothecoides. Plant Physiol. 2015, 167:586-599.

49. Liu Z, Gao Y, Chen J, Imanaka T, Bao J, Hua Q: Analysis of metabolic fluxes for better understanding of mechanisms related to lipid accumulation in oleaginous yeast Trichosporon cutaneum. Bioresour. Technol. 2013, 130:144151.

50. Hamilton ML, Haslam RP, Napier J a., Sayanova O: Metabolic engineering of Phaeodactylum tricornutum for the enhanced accumulation of omega-3 long chain polyunsaturated fatty acids. Metab. Eng. 2014, 22:3-9.

51. Jiang M, Guo B, Wan X, Gong Y, Zhang Y, Hu C: Isolation and characterization of the diatom Phaeodactylum $\Delta 5$-elongase gene for transgenic LC-PUFA production in Pichia pastoris. Mar. Drugs 2014, 12:1317-1334.

52. Tai M, Stephanopoulos G: Engineering the push and pull of lipid biosynthesis in oleaginous yeast Yarrowia lipolytica for biofuel production. Metab. Eng. 2013, 15:1-9.

53. Yao Y, Lu Y, Peng K-T, Huang T, Niu Y-F, Xie W-H, Yang W-D, Liu J-S, Li H-Y: Glycerol and neutral lipid production in the oleaginous marine diatom Phaeodactylum tricornutum promoted by overexpression of glycerol-3phosphate dehydrogenase. Biotechnol. Biofuels 2014, 7:110.

54. Trentacoste EM, Shrestha RP, Smith SR, Glé C, Hartmann AC, Hildebrand M, Gerwick WH: Metabolic engineering of lipid catabolism increases microalgal 
lipid accumulation without compromising growth. Proc. Natl. Acad. Sci. U. S. A. 2013, 110:19748-19753.

55. Daboussi F, Leduc S, Maréchal A, Dubois G, Guyot V, Perez-Michaut C, Amato A, Falciatore $A$, Juillerat $A$, Beurdeley $M$, et al.: Genome engineering empowers the diatom Phaeodactylum tricornutum for biotechnology. Nat. Commun. 2014, 5:3831.

56. Sander JD, Joung JK: CRISPR-Cas systems for editing, regulating and targeting genomes. Nat. Biotechnol. 2014, 32:347-355.

57. Stovicek V, Borodina I, Forster J: CRISPR-Cas system enables fast and simple genome editing of industrial Saccharomyces cerevisiae strains. Metab. Eng. Commun. 2015, 2:13-22.

58. Li Y, Lin Z, Huang C, Zhang Y, Wang Z, Tang Y, Chen T, Zhao X: Metabolic engineering of Escherichia coli using CRISPR-Cas9 meditated genome editing. Metab. Eng. 2015, 31:13-21.

59. Karas BJ, Diner RE, Lefebvre SC, McQuaid J, Phillips APR, Noddings CM, Brunson JK, Valas RE, Deerinck TJ, Jablanovic J, et al.: Designer diatom episomes delivered by bacterial conjugation. Nat. Commun. 2015, 6:6925.

60. Xu P, Li L, Zhang F, Stephanopoulos G, Koffas M: Improving fatty acids production by engineering dynamic pathway regulation and metabolic control. Proc. Natl. Acad. Sci. U. S. A. 2014, 111:11299-11304.

61. Kitcha S, Cheirsilp B: Enhanced lipid production by co-cultivation and coencapsulation of oleaginous yeast Trichosporonoides spathulata with microalgae in alginate gel beads. Appl. Biochem. Biotechnol. 2014, 173:522534.

62. Wrede D, Taha M, Miranda AF, Kadali K, Stevenson T, Ball AS, Mouradov A: Cocultivation of fungal and microalgal cells as an efficient system for harvesting microalgal cells, lipid production and wastewater treatment. PLoS One 2014, 9:e113497.

63. Silaban A, Bai R, Gutierrez-Wing MT, Negulescu II, Rusch KA: Effect of organic carbon, $\mathrm{C}: \mathrm{N}$ ratio and light on the growth and lipid productivity of microalgae/cyanobacteria coculture. Eng. Life Sci. 2014, 14:47-56.

64. Ge S, Usack J, Spirito CM, Angenent LT: Long-term n-caproic acid production from yeast-fermentation beer in an anaerobic bioreactor with continuous product extraction. Environ. Sci. Technol. 2015, 49:8012-8021. 
65. Orth JD, Thiele I, Palsson BØ: What is flux balance analysis? Nat. Biotechnol. 2010, 28:245-248.

66. Machado D, Herrgård M: Systematic evaluation of methods for integration of transcriptomic data into constraint-based models of metabolism. PLOS Comput. Biol. 2014, 10:e1003580.

67. Ye C, Xu N, Chen H, Chen YQ, Chen W, Liu L: Reconstruction and analysis of a genome-scale metabolic model of the oleaginous fungus Mortierella alpina. BMC Syst. Biol. 2015, 9:1.

68. Ranganathan S, Tee TW, Chowdhury A, Zomorrodi AR, Yoon JM, Fu Y, Shanks J V., Maranas CD: An integrated computational and experimental study for overproducing fatty acids in Escherichia coli. Metab. Eng. 2012, 14:687-704.

69. Burgard AP, Pharkya $P$, Maranas $C D$ : OptKnock: A bilevel programming framework for identifying gene knockout strategies for microbial strain optimization. Biotechnol. Bioeng. 2003, 84:647-657.

70. Ranganathan S, Suthers PF, Maranas CD: OptForce: An optimization procedure for identifying all genetic manipulations leading to targeted overproductions. PLoS Comput. Biol. 2010, 6:e1000744.

71. Ip K, Donoghue N, Kim MK, Lun DS: Constraint-based modeling of heterologous pathways: Application and experimental demonstration for overproduction of fatty acids in Escherichia coli. Biotechnol. Bioeng. 2014, 111:2056-2066.

72. Zomorrodi AR, Suthers PF, Ranganathan S, Maranas CD: Mathematical optimization applications in metabolic networks. Metab. Eng. 2012, 14:672686.

73. Chan WH, Mohamad MS, Deris S, Illias RM: A review of computational approaches for in silico metabolic engineering for microbial fuel production. Curr. Bioinform. 2013, 8:253-258.

74. Tan J, Zuniga C, Zengler K: Unraveling interactions in microbial communities from co-cultures to microbiomes. J. Microbiol. 2015, 53:295-305. 
Figure 1
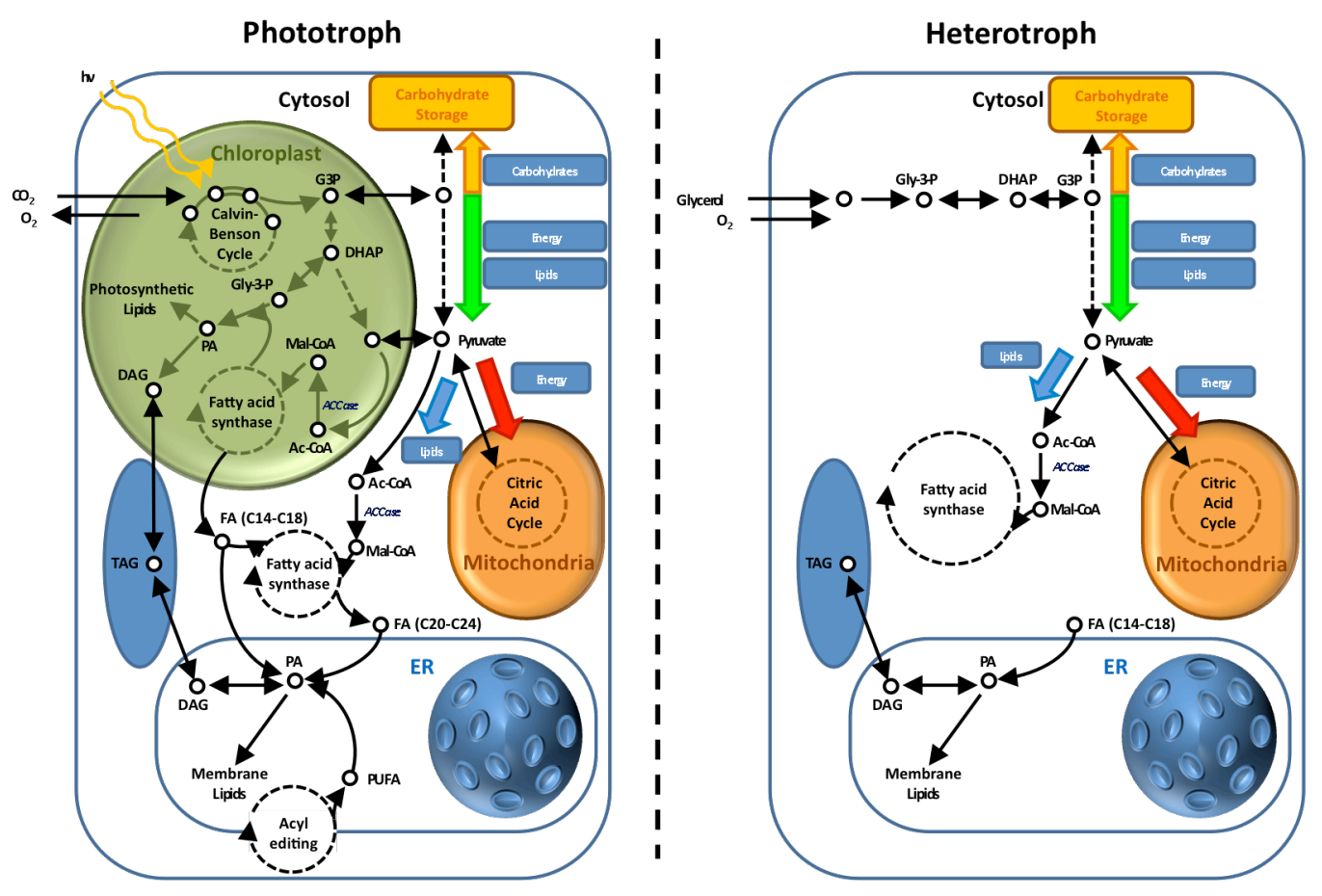


\section{Figure 2}

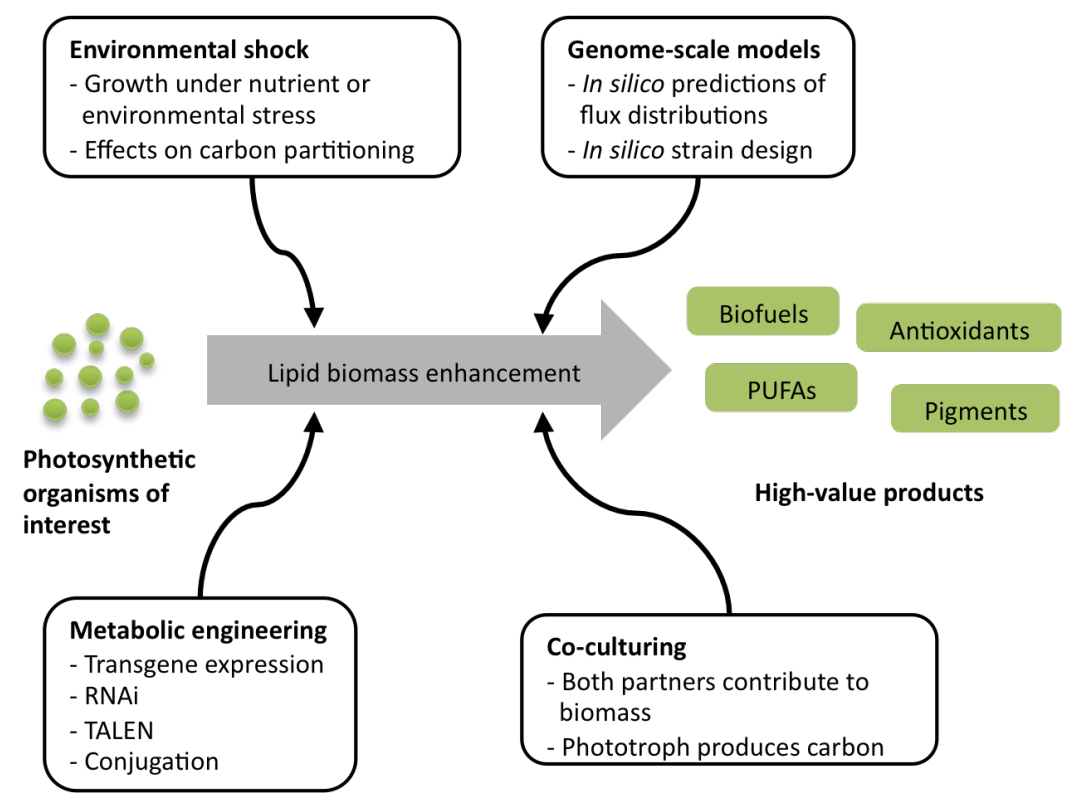


Figure 3

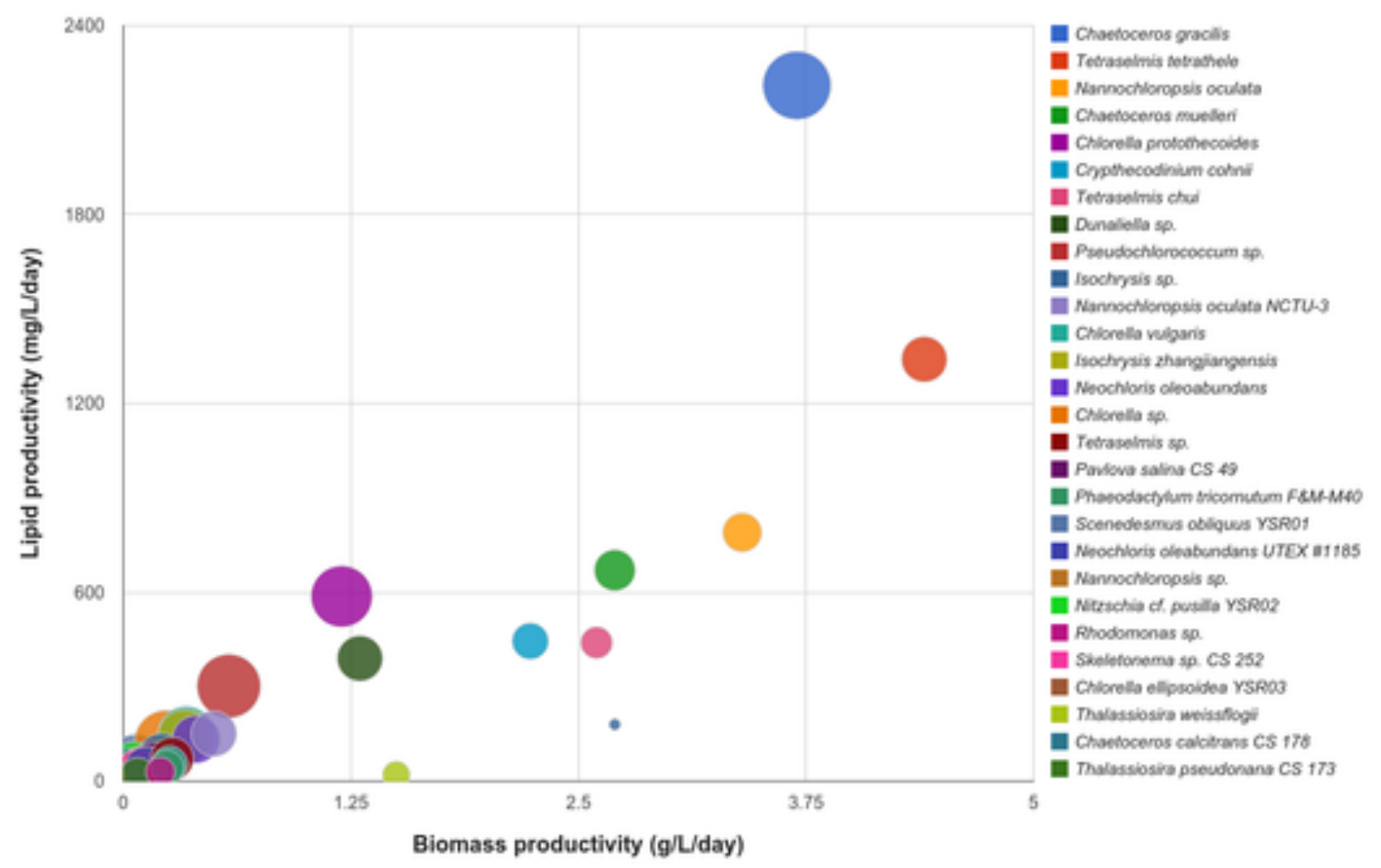

